\title{
TI.34.3
}

\section{Baseline Expectations for Trust in Federation Version 2}

- PDF: TI.34.3-BaselineExpectations-v2-2020-11.pdf

- Text: TI.34.3-BaselineExpectations-v2-2020-11.txt

\section{More Information}

\begin{tabular}{|l|l|}
\hline Repository ID & TI.34.3 \\
\hline Persistent URL & http://doi.org/10.26869/TI.34.3 \\
\hline Title & Baseline Expectations for Trust in Federation Version 2 \\
\hline Authors & Members of the InCommon Community Trust and Assurance Board \\
\hline Sponsor & InCommon Community Trust and Assurance Board (CTAB) \\
\hline Review & \\
\hline Status & Preserve \\
\hline Publish Date & November, 2020 \\
\hline DOI & $10.26869 /$ TI.34.3 \\
\hline Signature & \\
\hline Deprecated & No \\
\hline Future Review & November 2021 \\
\hline Supersedes & TI.34.2 http://doi.org/10.26869/TI.34.2 \\
\hline Format & PDF, Text \\
\hline Related Docs & \\
\hline Development Location & \\
\hline IP Framework & CC-BY 4.0 \\
\hline Subject Tags & InCommon, federation, assurance, trust, framework \\
\hline Notes & \\
\hline & \\
\hline
\end{tabular}

